\title{
Predictors of treatment dropout in self-guided web-based interventions for depression: An "individual patient data" meta-analysis
}

E. Karyotaki ${ }^{1,2 *}$, A. Kleiboer ${ }^{1,2}$, F. Smit ${ }^{1,2,3}$, D. T. Turner ${ }^{1,2}$, A. Mira Pastor ${ }^{4}$, G. Andersson $^{5,6}$, T. Berger ${ }^{7}$, C. Botella ${ }^{4}$, J. M. Breton ${ }^{4}$, P. Carlbring ${ }^{8}$, H. Christensen, E ${ }^{9}$. de Graaf $^{10,11}$, K. Griffiths ${ }^{12}$, T. Donker ${ }^{1,2}$, L. Farrer ${ }^{12}$, M. Huibers ${ }^{1,2}$, J. Lenndin ${ }^{13}$, A. Mackinnon $^{14}$, B. Meyer ${ }^{15,16}$, S. Moritz ${ }^{17}$, V. Spek ${ }^{18}$, K. Vernmark ${ }^{13,19}$ and P. Cuijpers ${ }^{1,2}$

1

Department of Clinical psychology, Vu University Amsterdam, Amsterdam, The Netherlands

${ }_{3}$ EMGO, Institute of Health Care Research, Vu University Medical Centre, Amsterdam, The Netherlands

3

${ }_{5}$ Department of Psychology and Technology, Jaume University, Castellon, Spain

5 Department of Behavioural Sciences and Learning, Sweden Institute for Disability Research, Linköping University, Sweden

${ }_{7}^{6}$ Department of Clinical Neuroscience, Psychiatry Section, Karolinska Institute for Disability Research, Stockholm, Sweden

Department of Clinical Psychology and Psychotherapy, University of Bern, Bern, Switzerland

${ }_{9}$ Department of Psychology, Stockholm University, Stockholm, Sweden

Black Dog Institute and University of New South Wales, Prince of Wales Hospital, Sydney, Australia

${ }_{11}$ Department of Clinical Psychological Science, Faculty of Psychology, Maastricht University, The Netherlands

11 Department of Medical Psychology \& Psychotherapy, Erasmus Medical Centre, Rotterdam, The Netherlands

${ }_{13}$ National Institute of Mental Health Research, The Australian National University, Sydney, Australia

Department of Behavioural Sciences and Learning, Linkoping University, Linkoping, Sweden

15

Research Department, Gaia AG, Hamburg, Germany

${ }_{17}$ Department of Psychology, City University, London, United Kingdom

${ }_{18}$ Department of Psychiatry and Psychotherapy, University Medical Centre Hamburg-Eppendorf, Hamburg, Germany

18 Avans Hogeschool, University of Tilburg, Tilburg, The Netherlands

19 Psykologpartners, Linkoping, Sweden

*Address for correspondence: Eirini Karyotaki, M.Sc. Res., Department of Clinical Psychology, VU University Amsterdam, Van der Boechorststraat 1, 1081 BT Amsterdam, The Netherlands

(Email: e.karyotaki@vu.nl) 


\section{ABSTRACT}

Background: It is well known that web-based interventions can be effective treatments for depression. However, dropout rates in web-based interventions are typically high, especially in self-guided web-based interventions where no coach or therapist is available. Rigorous empirical evidence regarding factors influencing dropout in self-guided web-based interventions is lacking due to small study sample sizes. In this paper we examined predictors of dropout in an individual patient data (IPD) meta-analysis to gain a better understanding of who may benefit from these interventions.

Method: A comprehensive literature search for all Randomized Controlled Trials (RCTs) of psychotherapy for adults with depression from 2006 to January 2013 was conducted. Then we approached authors to collect the primary data of studies of self-help web-based depression intervention trials. Predictors of dropout, such as socio-demographic, clinical, and intervention characteristics were examined.

Results: Data from 2,705 participants across ten RCTs of self-guided web-based interventions for depression were analysed. Almost $70 \%$ of the participants dropped out of the intervention before completing at least $75 \%$ of the treatment modules. The multivariate analysis indicated that male gender $(\mathrm{R} R=1.07)$, lower educational level $(\mathrm{RR}=1.22)$, CBTbased intervention $(R R=1.21)$ and comorbid anxiety symptoms $(R R=1.20)$ significantly increased the risk of dropping out, while for every additional four years of age, the risk of dropping out significantly decreased ( $\mathrm{RR}=.98)$.

Conclusions: Dropout can be predicted by several variables and is not randomly distributed. This knowledge may inform tailoring of online self-help interventions to prevent dropout in identified groups at risk.

Keywords: treatment, adherence, treatment dropout, self-help, web-based interventions, depression, eHealth

\section{INTRODUCTION}

A large body of research has suggested that web-based interventions can be effective treatments for depression with comparable effect sizes to face-to-face treatments (Andrews $e t$ al., 2010, Cuijpers et al., 2010, Spek et al., 2007a). However, meta-analytic studies have also shown that self-guided web-based interventions (i.e. interventions that patients work through on their own without guidance) show less promising results than guided web-based 
interventions that are delivered with support from a coach or therapist (Andersson and Cuijpers, 2009, Cuijpers et al., 2011, Gellatly et al., 2007, Richards and Richardson, 2012, Spek et al., 2007a). One explanation for the difference in effectiveness between guided and unguided web-based interventions is that the human support involved in guided interventions increases treatment adherence through accountability to a coach or therapist who is seen as trustworthy, benevolent, and an expert (Mohr et al., 2011). Furthermore, guided web-based interventions often not only involve a supportive coach who helps participants through the program but also more often than unguided interventions include human contact before treatment (e.g. during a diagnostic interview; Johansson and Andersson, 2012) or include referral by a therapist (Berger et al., 2011, Marks and Cavanagh, 2009), which may add to feelings of accountability.

In line with the idea of 'supportive accountability', higher dropout rates have been found in unguided web-based interventions for depression compared to guided web-based interventions with average levels of adherence estimated at $26 \%$ in unguided interventions and $72 \%$ in guided interventions (Richard and Richardson, 2012). In addition, empirical evidence has shown that greater exposure to the intervention is related to better treatment outcomes (Donkin et al., 2011) suggesting that efforts to increase adherence rates in webbased interventions may lead to better outcomes. To gain a better understanding of who may benefit from unguided web-based interventions and how we can improve adherence rates, there is a strong need to identify characteristics of individuals and interventions that are related to treatment dropout, as unguided interventions are much easier to implement and less costly than guided web-based interventions.

A few studies have already investigated this issue (Christensen et al., 2009, Waller and Gilbody, 2009). However, studies that have been conducted so far often lack the power to find reliable effects of predictors and moderators. In the current study we bring together the data from separate studies and employ a new strategy named Individual Patient Data (IPD) metaanalysis. IPD meta-analysis was developed to address research questions that require large sample sizes and is based on data pooled from individual RCTs (Bower et al., 2013). In this way it increases the power and precision to detect predictors and moderators. This study aimed to identify socio-demographic, clinical, and intervention characteristics that predict dropout rates in self-guided web-based interventions for depression. In the context of the present paper, the term adherence is defined as the percentage of treatment modules that were completed. Dropout rate was defined as a completion rate of less than seventy five percent of the intervention modules, as we considered that in most interventions the core treatment elements are administered in this part of the treatment. 


\section{METHOD}

\section{Search Strategy for identification and selection of studies}

We used an existing database of randomized trials of psychological treatments for depression. The database has already been used by several published meta-analyses (www.evidencebasedpsychotherapies.org) and its detailed description can be found elsewhere (Cuijpers et al., 2008). This database has been developed and is periodically updated by a comprehensive literature search of the following health related databases: Cochrane Central Register of control trials, PubMed, Psych Info and Embase from 1996 to January 2013. In these electronic searches, various key terms covering the concepts of psychotherapy and depression were used in different combinations (both MeSH-terms and text words). For a detailed description of the searches the reader is referred to Cuijpers et al. (2008). In addition, several systematic reviews and meta-analyses in this research field have been cross-checked throughout the development of this database in order to ensure that no trials were missing. Along with the use of this database, we contacted authors and asked them to provide us with access to the datasets of trials that were not yet published.

\section{Inclusion Criteria for studies}

We included (a) randomized controlled trials (RCTs), (b) comparing a psychological intervention (c) delivered through the web (d) without any form of personal guidance, (e) with a control or comparison group, (f) aimed at adults with depression (based on a clinical interview or on elevated depressive symptoms ratings on self-report measures).

\section{Quality Assessment}

The validity of the studies included in the present IPD meta-analysis was examined by two independent reviewers (E.K. and D.T.) according to four criteria of the Cochrane Risk of Bias assessment tool (Higgins and Green, [updated March 2011], Higgins JPT and Altman DG, 2008). We tested if the allocation concealment was adequately generated (sequence generation), the allocation was sufficiently concealed (allocation concealment), the knowledge of the allocated intervention was adequately prevented (blinding), and any incomplete outcome data were sufficiently addressed. However, we did not consider that incomplete outcome data could influence the results of the present IPD meta-analysis since the primary aim of this paper was to identify factors influencing treatment dropout. Finally, when the information that was provided in the papers did not provide sufficient information to assess quality, we contacted the primary authors to ask what procedure was actually followed and subsequently we ran sensitivity analysis based on what the papers reported. Disagreement 
between the reviewers was resolved through discussion, and if needed a third reviewer was consulted (P.C.).

\section{Data extraction and preparation}

Two authors independently extracted data included in the present meta-analysis (E.K. and D.T.). We first contacted authors of RCTs that satisfied the inclusion criteria and we asked them whether they would permit us access to their primary datasets. We identified the variables which were common to all or most of the included datasets. These were the following: randomized group (therapy or control), baseline and follow up depression scores, age, gender, educational level, employment status, relationship status (being in or not in a relationship), number of modules completed and presence of anxiety symptoms at baseline (yes/no; based on a clinical interview or on elevated anxiety symptoms ratings on self-report measures). Finally, we combined the individual datasets into one large pooled dataset.

\section{Statistical analysis}

In this paper, data were extracted only for intervention groups and not for control comparison conditions as we only looked at predictors of treatment adherence. Studies included in the present IPD meta-analysis used measures such as the CES-D or the BDI to monitor change in depression. These depression measures were standardized (transformed into z-scores) within each study before we conducted the main analysis. We also conducted sensitivity analyses to assess the impact of baseline severity on dropout from treatment for CES-D and BDI separately. We analysed the effects of predictors on dropout from treatment using a designbased analysis of the data to account for the clustering of participants within studies. Individual patient data were analysed by a poisson regression model for patients nested within studies to obtain relative risks (RR) of treatment dropout on the selected factors, adjusted for the other predictors in the Poisson model and taking into account the clustered data structure by obtaining robust (Hubert-White) standard errors based on the first-order Taylor-series linearization method as implemented in Stata version SE 12.1 (StataCorp., 2011). This methodological approach is computationally efficient in synthesizing and estimating the effect of predictors (Zou, 2004). We conducted the analysis in three steps. First we conducted a series of bivariate analyses to assess the RR of each factor at a time (the so called 'bivariate model'). Then we repeated the analyses with all factors simultaneously entered in the Poisson model (the so called 'complete model'). Lastly, we simplified the complete model by only retaining those factors in the model that were statistically significant by eliminating factors that were not significant (the 'parsimonious model'). Finally, we performed sensitivity analysis to assess the impact of the included studies' quality on dropout from treatment and 
we checked whether the assumption of linearity was met for the relationship between dependent and independent variables.

\section{RESULTS}

\section{Selection of included studies}

A total number of 14,164 abstracts were identified through bibliographic database searching. After the removal of duplicates, 10,474 abstracts were examined. A total of 1,476 full text papers were retrieved for potential inclusion. After the exclusion of 1,123 studies, 353 trials were included in the database. We searched through this database and in additional sources (grey literature, researchers on this field) and we identified 13 eligible RCTs for inclusion in the current meta-analysis. We were unable to retrieve the data from three studies (Clarke et al., 2005; Clarke et al., 2009; Clarke et al, 2002) and included 10 RCTs in the present IPD meta-analysis (77\%). Figure 1 presents the study selection process. Overall, the three studies that we did not include ( $n=302$ ) were very similar to the 10 included studies, except for the method of recruitment (all participants in these 3 studies were recruited through an HMO in the Unites States, while none of the other studies recruited patients in this way). The main outcome measures in these studies were the CES-D and the Patient Health Questionnaire-8 items (PHQ-8) (Clarke et al., 2005, Clarke et al., 2009, Clarke et al., 2002).

$$
=\text { Figure } 1=
$$

\section{Study Characteristics}

In the present IPD meta-analysis, ten studies with a total of 2,705 participants were included. All the examined studies recruited their participants from the community, and they were conducted across six different countries: Australia $(n=2)$, Germany $(n=2)$, Spain $(n=1)$, Sweden $(n=1)$, Switzerland $(n=1)$, and the Netherlands $(n=3)$. The majority of the included studies used self-report outcome measures for depression on which the participants needed to score above a predetermined cut-off point in order to be included in the trial. Seven out of the ten included studies used the BDI as a primary outcome measure while the remaining trials used the CES-D.

All included unguided web-based interventions were based on three different theoretical models of psychotherapy. The majority of the included studies used interventions based on CBT principles $(n=8)$. The remaining studies used either PST $(n=1)$ or they compared webbased CBT with IPT $(n=1)$. Table 1 shows selected characteristics of the included studies.

$$
=\text { Table } 1=
$$


Most of the participants were female ( $\mathrm{n}=1,945 / 2,705 ; 72 \%)$, aged between $25-34$ years $(\mathrm{n}=741 / 2,705 ; 27 \%)$, and educated at University level $(\mathrm{n}=1,933 / 2,705 ; 71 \%)$. The average score on the CES-D at baseline assessment was $35.5(\mathrm{SD}=11.5)$, while the average score on the BDI was $28.4(\mathrm{SD}=13.5)$ indicating a high degree of severity. The average score on the CES-D and the BDI reduced at the post-treatment assessment to $24.2(\mathrm{SD}=13.2 ; \mathrm{n}=650)$ and 20.7 ( $\mathrm{SD}=14.8 ; \mathrm{n}=495)$ respectively. The majority of the sample reported symptoms of comorbid anxiety $(\mathrm{n}=1,689 / 2,705 ; 71.6 \%)$ (Table 2$)$.

$$
=\text { Table } 2=
$$

\section{Quality Assessment}

All the included studies had acceptable methodological quality. The sequence was adequately generated, and the allocation was adequately concealed. Moreover, all trials used self-report outcome measures, which were administered via the Internet. Therefore, blinding of assessors was considered as adequately addressed across the ten studies of this IPD meta-analysis. However, the participants were not blinded to the interventions, and this may have caused bias. Finally, all included RCTs used intention-to-treat (ITT) analyses including all the randomized participants in their post treatment analyses, which indicates that incomplete outcome data were adequately addressed (see Table 1).

\section{Predictors of dropout in self-guided web-based treatment for depression}

The results of the bivariate analyses indicated that male gender $(\mathrm{RR}=1.05 ; 95 \% \mathrm{CI}: 1.01 \sim$ 1.11), participants with a low educational background ( $R R=1.23$; 95\% CI: $1.13 \sim 1.33$ ), the presence of comorbid anxiety symptoms (RR=1.18; 95\% CI: $1.01 \sim 1.38)$ and CBT-based interventions $(\mathrm{RR}=1.19 ; 95 \% \mathrm{CI}: 1.03 \sim 1.39)$ were related to a higher risk of dropping out. Finally, the chance of dropping out significantly decreased for every four years of age increase ( $\mathrm{RR}=.98 ; 95 \% \mathrm{CI}: .97 \sim 99)$. The remaining variables/potential predictors (baseline severity of depression, relationship status, number of intervention modules and employment status) failed to achieve a statistically significant level of $p<.05$ in the bivariate analysis (see table 3).

Additionally, under the parsimonious model, male gender ( $\mathrm{RR}=1.07$; 95\% CI: $1.02 \sim 1.14$ ), lower educational level ( $\mathrm{RR}=1.22,95 \% \mathrm{CI}$ : $1.09 \sim 1.36)$, CBT-based intervention $(\mathrm{RR}=1.21$; 95\% CI: $1.04 \sim 1.41)$, older age (RR=.98; 95\% CI: .97 .99) and comorbid anxiety (RR= 1.20; 95\%CI: $1.01 \sim 1.41)$ remained statistically significant predictors of dropout from treatment. However, depression severity, employment status and relationship status were nonsignificant after controlling for the other predictors (see table 3). The number of treatment 
modules was omitted from the multivariate analysis due to collinearity with type of intervention (the variable number of modules had a linear relationship with the type of intervention variable; Pearson's $r=-.3, \mathrm{p}<.001)$.

$$
=\text { Table } 3=
$$

\section{Sensitivity analysis}

We analysed the impact of depression severity on dropout for CES-D and BDI scores separately. Individuals who scored higher on CES-D at baseline had a higher risk of dropping out than those with lower scores $(\mathrm{RR}=1.004,95 \%$ CI 1.003 to 1.005, obs $=1987 \mathrm{p}<0.001)$. Separate analysis for BDI scores at baseline did not produce statistically significant results $(\mathrm{p}>0.05)$. However, the results of BDI analysis were based on a considerably smaller, though sufficiently powered, number of participants $(n=718)$.

Three studies did not report all relevant information regarding allocation concealment in the published papers (although personal contact with the primary authors illustrated that the allocation was adequately concealed) and thus, we decided to run sensitivity analysis based on what the papers reported. We examined the impact of quality of the included studies on treatment dropout. Study quality did not significantly predict treatment dropout ( $p>.05)$. Further, we controlled for study quality in our final parsimonious model. The predictors remained the same after adjusting for the quality of the included studies.

\section{DISCUSSION}

\section{Main findings}

The present IPD meta-analysis aimed to identify predictors of treatment dropout in selfguided web-based interventions for depression. We tested the relationship between dropout and several socio-demographic, clinical and intervention characteristics. The multivariate analysis of 2,705 individual patients' data revealed that being male; having attained a lower educational level; a younger age, completing self-guided web-based CBT (or an e-health intervention with about 8 sessions); and having comorbid anxiety symptoms significantly increased the risk of dropping out before the completion of $75 \%$ of treatment modules and thus were related to high treatment's dropout.

\section{Placing our findings in the wider context of the literature}

The finding that gender predicted treatment dropout has not been identified by previous literature. However, this result may reflect a different coping strategy between the two genders. Previous research has shown that females generally present with a higher effort to 
cope with depression compared to males (Babwah et al., 2006). These efforts might enhance their willingness to continue and complete web-based interventions without any form of guidance. There is also evidence to support the idea that women are generally more conscientious regarding health issues compared to men (Babwah et al., 2006). These differences in health attitudes could partly account for the differences in treatment compliance rates between the genders.

A lower educational background has also been identified as a risk for dropping out in previous research and it has been suggested that low educational status is a barrier to adherence to web-based CBT because of greater difficulties in understanding the intervention content and procedure and limited abilities in using information technology which may result in diminished motivation to continue and complete a self-guided web-based treatment (Waller and Gilbody, 2009).

Unlike the results from this study that showed that younger age was related to low treatment adherence, previous research showed that younger individuals had higher adherence to webbased treatment (Christensen, 2009).

No research has looked at the relation between different therapeutic models and treatment adherence. This study showed that individuals taking part in CBT-based interventions had a $23 \%$ higher risk of dropping out of treatment than individuals taking part in the PST and IPT interventions. It is possible that the implementation of CBT without any form of guidance is rather difficult. However, it is important to stress that in our analyses CBT was confounded by the number of sessions. Hence, not CBT but the larger number of sessions associated with CBT may very well be the real predictor of dropout. This would be in accordance with previous literature. Christensen et al. (2009) found that dropout progressively increases with the length of the intervention.

Comorbid anxiety symptoms increased the risk of dropping out of the treatment 16 per cent. It is important, however, to stress that studies included in this meta-analysis were not designed for the treatment of anxiety or to deal with comorbid anxiety and therefore the reason for this finding is unclear. Further research is needed to clarify this.

None of the remaining variables significantly predicted treatment attrition and results derived by the present IPD meta-analysis were not influenced by quality of the included studies. The lack of a significant effect on adherence of relationship status is consistent with results reported by Christensen et al. (2009). Further, Christensen et al. (2009) concluded that dropout increases with the severity of baseline depression. The findings from the present study suggest that the severity of depression does not significantly predict dropout from 
treatment. However, when we examined the impact of baseline severity separately for CES-D and BDI we found a significant but small higher risk for dropping out of treatment for patients who scored higher on CES-D at baseline, a result which is consistent with the conclusions of Christensen et al. (2009).

\section{Strengths and limitations}

One of the strengths of the present IPD meta-analysis was that it was based on a novel methodological approach that it is considered a gold standard for identifying predictors, moderators and mediators to treatment dropout and outcome. Combining raw individual data from several studies into one single dataset provides adequate power and precision to detect predictors of treatment attrition. Further, the systematic literature search employed by the present IPD meta-analysis reduced the risk of introducing study selection bias into the results.

In spite of the aforementioned strengths it should be noted that the present study has several limitations. Among these limitations was the risk of availability bias. We could access ten RCTs' individual patient datasets out of thirteen eligible studies. Although this is higher than in other IPD meta-analyses (Riley et al., 2007), the ten available RCTs might differ in several ways from the three unavailable studies. Moreover, some of the predictor variables were not reported across all the ten RCTs. This might have resulted in lower power to predict effects for some of the variables of interest, although the IPD was better powered to detect a true effect than a single trial. Further, such small effects would be less relevant from a clinical or public health perspective. IFinally, the participants of the present IPD meta-analysis differ from patients in clinical samples. For instance, all the participants were recruited through the community and were proactively seeking help for their symptoms. Thus, the present findings might not be generalized to the whole population with depression but it is representative for help seeking individuals in the community.

All these predictors should be taken into account in future development of self-guided interventions for depression. For example, different features of web-based interventions may be appealing to different individuals and it is important to find out what works best for whom. Future interventions could, for example, employ more audio-visual components such as videos or gaming and less written material for individuals with a lower education. This knowledge will help in utilizing the self-guided form of web-based interventions in the most efficient and effective way. Future studies may need to be tailored to the particular needs of individuals with comorbid anxiety symptoms, male gender, with a low educational background and young age. Further, future research should also examine dropout at different time points, as treatment dropout in different time intervals may represent different processes. Other psychological predictors such as personality styles, motivation and preferences should 
be included in future trials to inform tailoring. This might prevent dropout in future versions of self-guided web based interventions.

\section{Acknowledgments}

The authors would like to thank Erica Weitz, Spyridon Kolovos and Ioannis Gonnianakis for assistance in various parts of this IPD meta-analysis.

\section{Financial Support}

This research received no specific grant from any funding agency, commercial or not-forprofit sectors.

\section{Conflict of interest}

None

\section{Ethical standards}

The authors assert that all procedures contributing to this work comply with the ethical standards of the relevant national and institutional committees on human experimentation and with the Helsinki Declaration of 1975, as revised in 2008. 


\section{References}

Andersson, G. \& Cuijpers, P. (2009). Internet-based and other computerized psychological treatments for adult depression: a meta-analysis. Cogn Behav Ther 38, 196-205.

Andrews, G., Cuijpers, P., Craske, M. G., McEvoy, P. \& Titov, N. (2010).

Computer therapy for the anxiety and depressive disorders is effective, acceptable and practical health care: a meta-analysis. PLoS One 5, e13196.

Babwah, F., Baksh, S., Blake, L., Cupid-Thuesday, J., Hosein, I., Sookhai, A., Poon-King, C. \& Hutchinson, G. (2006). The role of gender in compliance and attendance at an outpatient clinic for type 2 diabetes mellitus in Trinidad. Rev Panam Salud Publica 19, 79-84.

Berger, T., Caspar, F., Richardson, R., Kneubuhler, B., Sutter, D. \& Andersson, G. (2011). Internet-based treatment of social phobia: a randomized controlled trial comparing unguided with two types of guided self-help. Behav Res Ther 49, 158-69.

Bower, P., Kontopantelis, E., Sutton, A., Kendrick, T., Richards, D. A., Gilbody, S., Knowles, S., Cuijpers, P., Andersson, G., Christensen, H., Meyer, B., Huibers, M., Smit, F., van Straten, A., Warmerdam, L., Barkham, M., Bilich, L., Lovell, K. \& Liu, E. T. (2013). Influence of initial severity of depression on effectiveness of low intensity interventions: meta-analysis of individual patient data. BMJ 346, f540.

Christensen, H., Griffiths, K. M. \& Farrer, L. (2009). Adherence in internet interventions for anxiety and depression. J Med Internet Res 11, e13.

Clarke, G., Eubanks, D., Reid, E., Kelleher, C., O'Connor, E., DeBar, L. L., Lynch, F., Nunley, S. \& Gullion, C. (2005). Overcoming Depression on the Internet (ODIN) (2): a randomized trial of a self-help depression skills program with reminders. $J$ Med Internet Res 7, e16.

Clarke, G., Kelleher, C., Hornbrook, M., Debar, L., Dickerson, J. \& Gullion, C. (2009). Randomized effectiveness trial of an Internet, pure self-help, cognitive behavioral intervention for depressive symptoms in young adults. Cogn Behav Ther 38, 222-34.

Clarke, G., Reid, E., Eubanks, D., O'Connor, E., DeBar, L. L., Kelleher, C., Lynch, F. \& Nunley, S. (2002). Overcoming depression on the Internet (ODIN): a randomized controlled trial of an Internet depression skills intervention program. $J$ Med Internet Res 4, E14.

Cuijpers, P., Donker, T., Johansson, R., Mohr, D. C., van Straten, A. \& Andersson, G. (2011). Self-guided psychological treatment for depressive symptoms: a meta-analysis. PLoS One 6, e21274.

Cuijpers, P., Donker, T., van Straten, A., Li, J. \& Andersson, G. (2010). Is guided self-help as effective as face-to-face psychotherapy for depression and anxiety 
disorders? A systematic review and meta-analysis of comparative outcome studies. Psychol Med 40, 1943-57.

Cuijpers, P., van Straten, A., Warmerdam, L. \& Andersson, G. (2008).

Psychological treatment of depression: a meta-analytic database of randomized studies. BMC Psychiatry 8, 36.

de Graaf, L. E., Gerhards, S. A., Arntz, A., Riper, H., Metsemakers, J. F., Evers, S. M., Severens, J. L., Widdershoven, G. \& Huibers, M. J. (2009). Clinical effectiveness of online computerised cognitive-behavioural therapy without support for depression in primary care: randomised trial. Br J Psychiatry 195, 73-80.

de Graaf, L. E., Gerhards, S. A., Arntz, A., Riper, H., Metsemakers, J. F., Evers, S. M., Severens, J. L., Widdershoven, G. \& Huibers, M. J. (2011). One-year follow-up results of unsupported online computerized cognitive behavioural therapy for depression in primary care: A randomized trial. J Behav Ther Exp Psychiatry 42, 89-95.

Donker, T., Bennett, K., Bennett, A., Mackinnon, A., van Straten, A., Cuijpers, P., Christensen, H. \& Griffiths, K. M. (2013). Internet-delivered interpersonal psychotherapy versus internet-delivered cognitive behavioral therapy for adults with depressive symptoms: randomized controlled noninferiority trial. J Med Internet Res 15, e82.

Donkin, L., Christensen, H., Naismith, S. L., Neal, B., Hickie, I. B. \& Glozier, N. (2011). A systematic review of the impact of adherence on the effectiveness of etherapies. J Med Internet Res 13, e52.

Farrer, L., Christensen, H., Griffiths, K. M. \& Mackinnon, A. (2011). Internetbased CBT for depression with and without telephone tracking in a national helpline: randomised controlled trial. PLoS One 6, e28099.

Gellatly, J., Bower, P., Hennessy, S., Richards, D., Gilbody, S. \& Lovell, K. (2007). What makes self-help interventions effective in the management of depressive symptoms? Meta-analysis and meta-regression. Psychol Med 37, 1217-28.

Higgins, J. \& Green, S. ([updated March 2011]). Cochrane Handbook for Systematic Reviews of Interventions : Version 5.1.0. The Cochrane collaboration.

Higgins JPT \& Altman DG (2008). Assessing risk of bias in included studies. In Cochrane handbook for systematic reviews of interventions (ed. Higgins JPT and Green S), pp. 187-241. Wiley.

Marks, I. \& Cavanagh, K. (2009). Computer-aided psychological treatments: evolving issues. Annu Rev Clin Psychol 5, 121-41.

Meyer, B., Berger, T., Caspar, F., Beevers, C. G., Andersson, G. \& Weiss, M. (2009). Effectiveness of a novel integrative online treatment for depression (Deprexis): randomized controlled trial. J Med Internet Res 11, e15. 
Mohr, D. C., Cuijpers, P. \& Lehman, K. (2011). Supportive accountability: a model for providing human support to enhance adherence to eHealth interventions. J Med Internet Res 13, e30.

Moritz, S., Schilling, L., Hauschildt, M., Schroder, J. \& Treszl, A. (2012). A randomized controlled trial of internet-based therapy in depression. Behav Res Ther 50, 513-21.

Richards, D. \& Richardson, T. (2012). Computer-based psychological treatments for depression: a systematic review and meta-analysis. Clin Psychol Rev 32, 329-42.

Riley, R. D., Simmonds, M. C. \& Look, M. P. (2007). Evidence synthesis combining individual patient data and aggregate data: a systematic review identified current practice and possible methods. J Clin Epidemiol 60, 431-9.

Spek, V., Cuijpers, P., Nyklicek, I., Riper, H., Keyzer, J. \& Pop, V. (2007a). Internet-based cognitive behaviour therapy for symptoms of depression and anxiety: a meta-analysis. Psychol Med 37, 319-28.

Spek, V., Nyklicek, I., Cuijpers, P. \& Pop, V. (2008). Predictors of outcome of group and internet-based cognitive behavior therapy. J Affect Disord 105, 137-45.

Spek, V., Nyklicek, I., Smits, N., Cuijpers, P., Riper, H., Keyzer, J. \& Pop, V. (2007b). Internet-based cognitive behavioural therapy for subthreshold depression in people over 50 years old: a randomized controlled clinical trial. Psychol Med 37, 1797-806.

StataCorp. (2011). Stata Statistical Software: Release 12.College Station. StataCorp LP.

Vernmark, K., Lenndin, J., Bjarehed, J., Carlsson, M., Karlsson, J., Oberg, J., Carlbring, P., Eriksson, T. \& Andersson, G. (2010). Internet administered guided self-help versus individualized e-mail therapy: A randomized trial of two versions of CBT for major depression. Behav Res Ther 48, 368-76.

Waller, R. \& Gilbody, S. (2009). Barriers to the uptake of computerized cognitive behavioural therapy: a systematic review of the quantitative and qualitative evidence. Psychol Med 39, 705-12.

Zou, G. (2004). A modified poisson regression approach to prospective studies with binary data. Am J Epidemiol 159, 702-6. 
Records identified through search in Cochrane Central Register, PubMed, and Embase ( $N=14164)$

Additional records identified through other sources (prior meta-analyses, contact with authors) $(\mathrm{N}=2)$

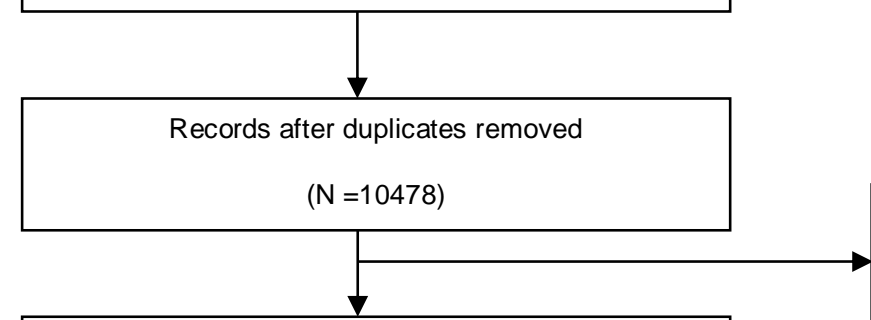

Full-text articles assessed for eligibility

$$
(N=1480)
$$

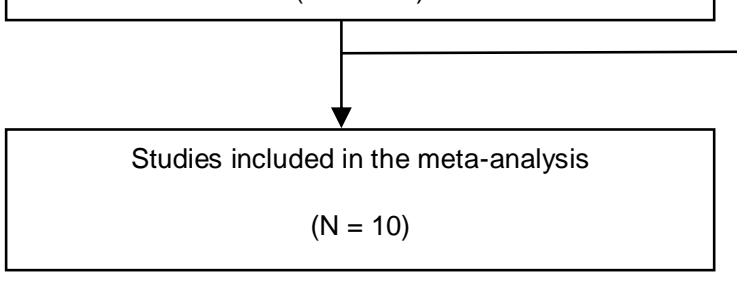

Records excluded

$$
(N=8998)
$$

Records excluded ( $N=1473)$

Studies with adolescents $(\mathrm{N}=74)$

Duplicate publication ( $\mathrm{N}=271)$

Not only depression ( $N=191)$

No psychotherapy $(\mathrm{N}=173)$

No random assignment $(\mathrm{N}=56)$

Maintenance trial $(\mathrm{N}=97)$

No self-guided web-based ( $N=599$ )

Not available $(\mathrm{N}=3)$

Figure 1. Studies selection process 
Table 1. Characteristics of included studies

\begin{tabular}{|c|c|c|c|c|c|c|c|}
\hline Study & Inclusion Criteria & $\mathrm{N}$ & $\begin{array}{l}\text { Outcome } \\
\text { Measure }\end{array}$ & $\begin{array}{l}\text { N. of } \\
\text { Modules }\end{array}$ & Intervention & Quality Ass.* & Country \\
\hline Berger et al. (2011) & BDI-II>13, MDD (Mini-DIPS) & 25 & BDI-II & 10 & CBT & ++++ & Switzerland \\
\hline $\begin{array}{l}\text { Botella et al. (under } \\
\text { submission) }\end{array}$ & $18-65$ years old. $B D I-I I$ not $>28$ & 36 & BDI-II & 8 & CBT & +++ & Spain \\
\hline $\begin{array}{l}\text { De Graaf et al. (2009, } \\
\text { 2011) }\end{array}$ & 8-65 years old, BDI score $\geq 16$ & 100 & BDI-II & 8 & CBT & ++++ & The Netherlands \\
\hline Donker et al. (2013) & CES-D $\geq 27$ & 1864 & CES-D & 4 & CBT, IPT & +++ & Australia \\
\hline Farrer et al. (2011) & $\mathrm{K} 10>20$ & 38 & CES-D & 5 & CBT & +++ & Australia \\
\hline $\begin{array}{l}\text { Kleiboer et al. (under } \\
\text { submission) }\end{array}$ & $35>C E S-D>16 ; 15>H A D S>8$ & 107 & CES-D & 5 & PST & +++ & The Netherlands \\
\hline Meyer et al. (2009) & Depression (BDI) & 320 & BDI & 10 & CBT & ++++ & Germany \\
\hline Moritz et al. (2012) & 18-65 years old, depression (BDI) & 105 & $\mathrm{BDI}$ & 10 & CBT & +++ & Germany \\
\hline Spek et al $(2008,2007 \mathrm{~b})$ & $50-57$ years old, EDS>12 & 102 & BDI-II & 10 & CBT & +++ & The Netherlands \\
\hline Vernmark et al. (2010) & MDD (SCID-I-CV) & 24 & BDI & 8 & CBT & ++++ & Sweden \\
\hline
\end{tabular}

BDI: Beck Depression Inventory; C: no internet intervention, supported by coach at pre agreed times; CBT: Cognitive and Behavioural Therapy; CES-D: Centre of Epidemiological Studies for Depression Scale; EDS: The Endiburg Depression Scale; HADS: Hospital Anxiety and Depression Scale; IPT: Interpersonal Psychotherapy; K10: The Kessler psychological distress scale; MDD: Major Depressive Disorder; Mini DIPS: Mini Diagnostic Interview for Psychiatric Disorders; N: Number; Quality Ass. : Quality Assessment; SCID-I-CV: Structural Clinical Interview for DSM-IV axis I disorders; PST: Problem Solving Therapy

* A positive or a negative sign is given in this column for the following quality criteria respectively: allocation sequence, allocation concealment, blinding of assessors, and incomplete outcome data (whether or not the study used intention-to-treat analysis) 
Mąblescribthographic and clinical characteristics of the included sample -Click here to download Table(s): Table 2.pelf

\section{Characteristics}

Age, $25-34$ years old $n(\%)$

Gender, females' n (\%)

CES-D at the baseline, mean (SD)

$\mathrm{BDI}$ at the baseline, mean (SD)

CES-D at post-treatment, mean (SD)

$\mathrm{BDI}$ at post-treatment, mean (SD)

Comorbid Anxiety, n (\%)
$741 / 2705(27)$

$1945 / 2705(72)$

$35.5(11.5)$

$28.4(13.5)$

$24.2(13.2)$

$20.7(14.8)$

1689/2705 (71.6)

BDI: Beck Depression Inventory; CES-D: Centre of Epidemiological studies for depression scale; n: number of patients; SD: Standard Deviation 
Malfale ${ }^{3}$-Pfedictors to self-guided web-based psychotherapy for depression

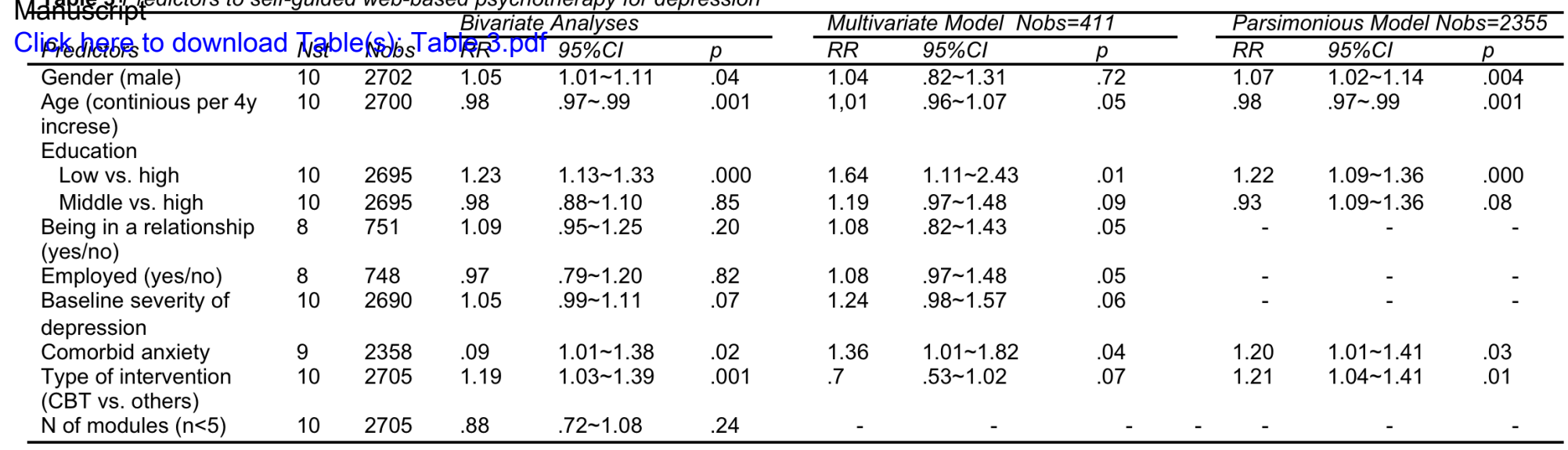

CBT vs. other: Cognitive Behavioural Therapy compared to other types of psychotherapy; Cl: Confidence Intervals; comp.: compare; $\mathrm{N}$ of modules: number of modules; Nobs: number of observations; Nst: number of studies; p: p-value; RR: Risk Ratio; Substandard Error; y: years; 